\title{
SOUTHERN HIGH-VELOCITY STARS
}

H.J. Augensen and W. Buscombe

Northwestern University

Using the model of the Galaxy presented by Eggen, LyndenBell and Sandage (1962), plane galactic orbits have been calculated for 800 southern high-velocity stars which possess parallax, proper motion, and radial velocity data. The stars with trigonometric parallaxes were selected from Buscombe and Morris (1958), supplemented by more recent spectroscopic data. Photometric parallaxes from infrared color indices were used for bright red giants studied by Eggen (1970), and for red dwarfs for which Rodgers and Eggen (1974) determined radial velocities. A color-color diagram based on published values of $(U-B)$ and $(B-V)$ for most of these stars is shown in Fig. 1. Plots of each of the orbital parameters apo- and perigalacticum, eccentricity, change in radial angle per cycle, radial and azimuthal periods, and instantaneous orbital inclination to the galactic plane versus color indices (U-B), $(B-V)$, and the ultraviolet excess $\delta(U-B)$ were also produced. In general, stars (especially $F$ dwarfs) with greater space velocities and also greater components of speed perpendicular to the galactic plane have larger ultraviolet excesses, eccentricities and inclinations of the orbits around the galactic center (Fig. 2). Also, a comparison is made between the HR diagrams for the southern high-velocity star group and that of M3, a globular cluster, and again for M67, an old open cluster. The high-velocity star group is found to resemble an old open cluster more than a globular cluster (Fig. 3). 


\section{REFERENCES}

Buscombe, W. and Morris, P.M. (1958). Mem. Mt. Stromlo Obs. 14. Eggen, O.J. and Stokes, N.R. (1970). Astrophys. J. 161, 199. Eggen, O.J., Lynden-Bell, D. and Sandage, A.R. (1962). Astrophys. Rodgers, $\frac{\text { J. }}{136}, 748$. and Eggen, O.J. (1974). Publ. Astron. Soc. Pacific $86,428$.

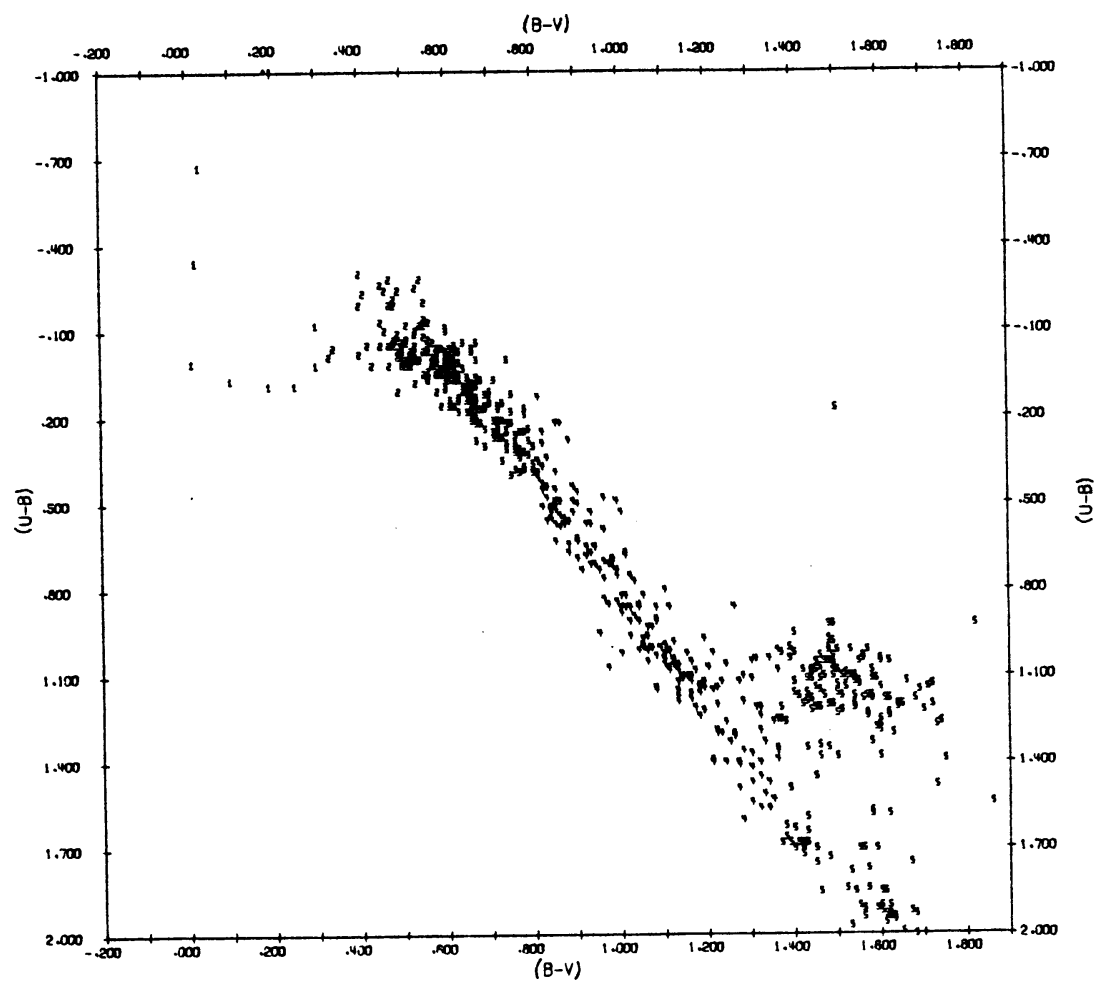

Fig. 1. A plot of (U-B) versus (B-V) for the southern high-velocity star group. Each symbol represents an approximate temperature class: $1=\mathrm{A}, 2=\mathrm{F}, 3=\mathrm{G}, 4=\mathrm{K}, 5=\mathrm{M}$. 


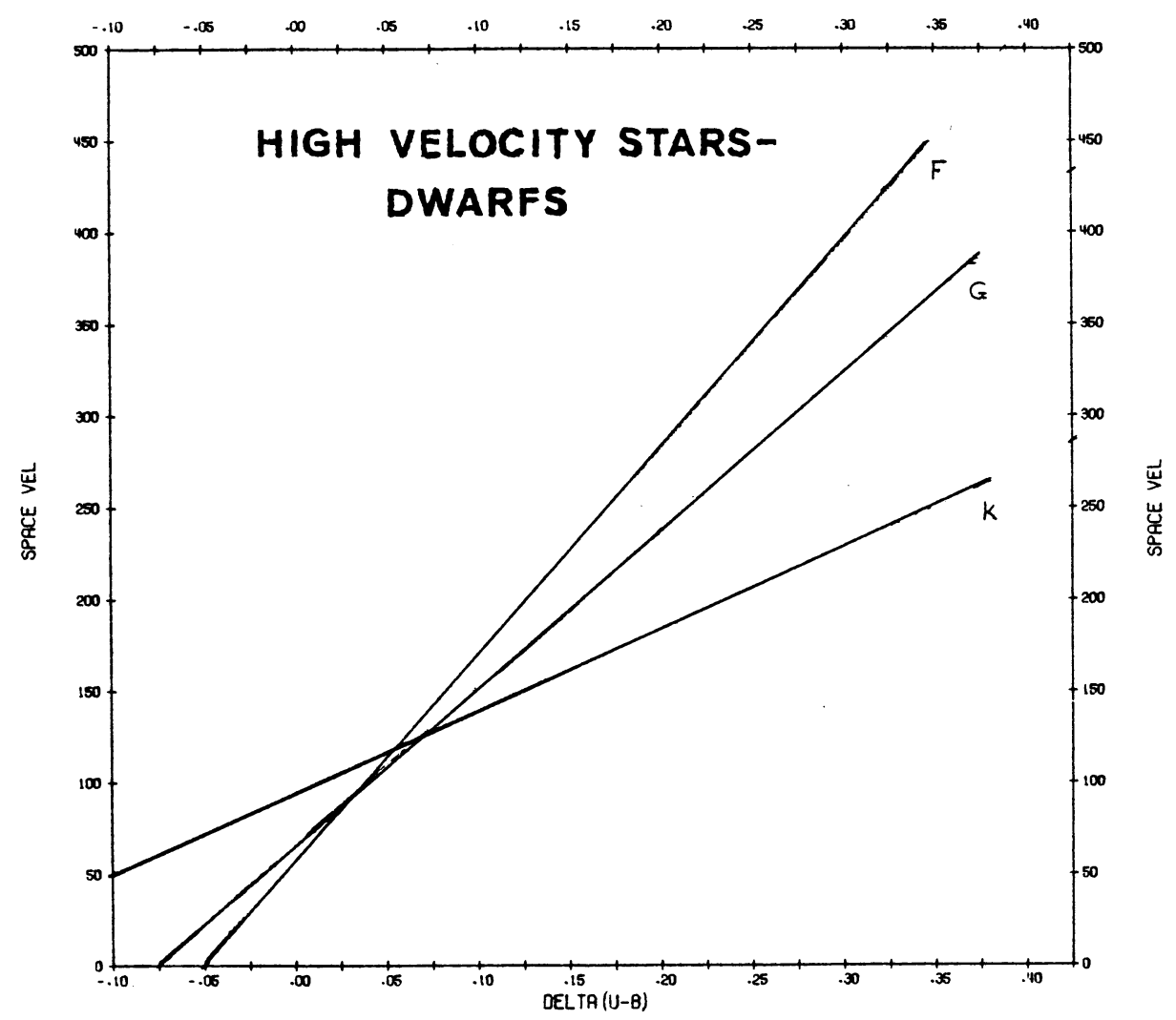

Fig. 2. A graph of the total space velocity versus the ultraviolet excess for $\mathrm{F}, \mathrm{G}$ and $\mathrm{K}$ high-velocity dwarf stars. At least squares fit to the data is displayed for each group. 


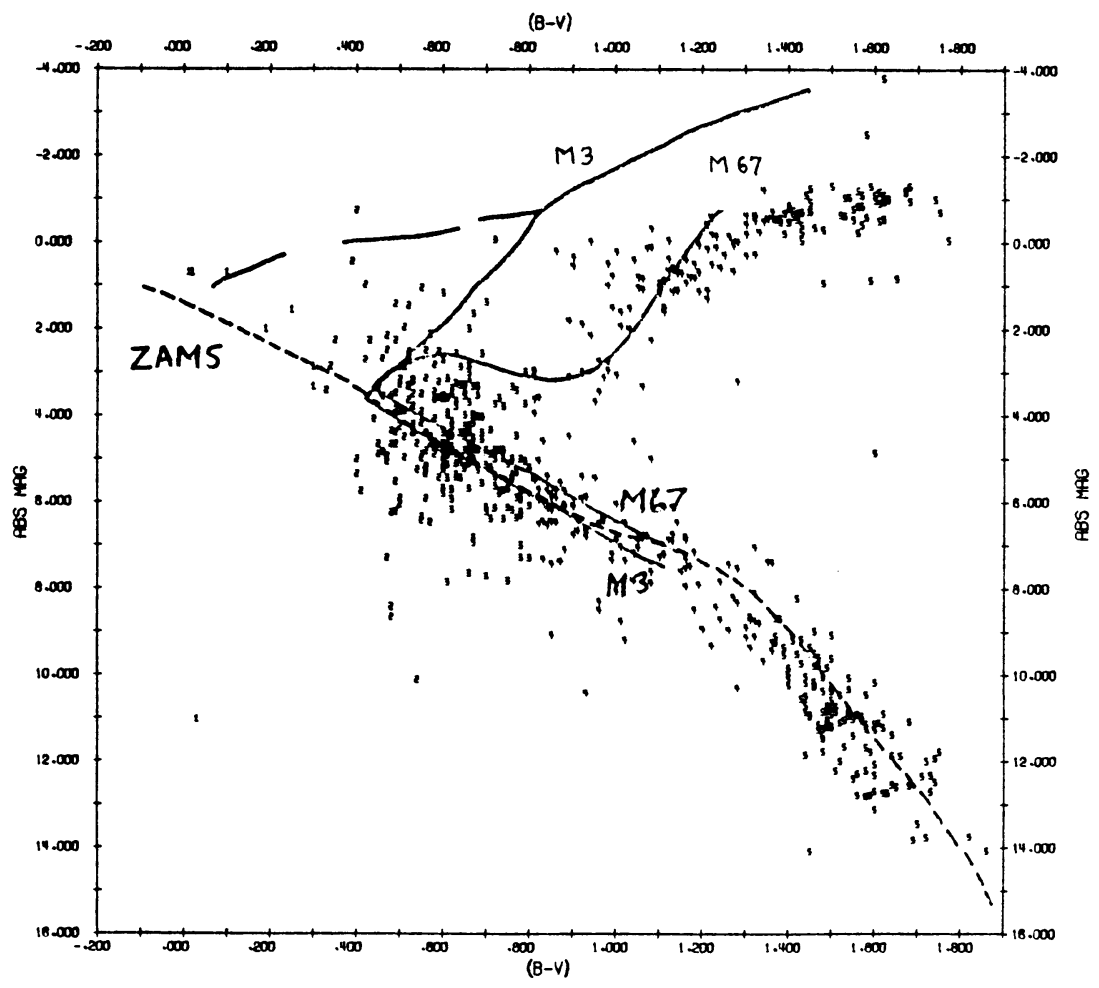

Fig. 3. The HR plot for the southern high-velocity star group. Superposed upon this diagram are sketches of the HR diagrams for M3, M67 and the zero-age main sequence. 